\title{
Substrate-Integrated-Waveguide Diplexer Filter for SATCOM-on-the-Move
}

\author{
Kamil Yavuz Kapusuz, Giani Ollivier, Jules Noppe, Jaron Van Maele, Sam Lemey and Hendrik Rogier, \\ IDLab-Electromagnetics group, imec-Ghent University, KamilYavuz.Kapusuz@UGent.be
}

\begin{abstract}
Satellite communication (SATCOM) on-the-move applications require low-cost compact antenna arrays to enable mobile users to track the satellites. Full-duplex operation requires efficient diplexers that satisfy stringent requirements in a compact footprint to enable tight integration within the antenna system. In this contribution, substrate-integrated-waveguide technology is leveraged to design a single-layer third-order diplexer filter based on a dual-mode cavity. Simulations and measurements demonstrate that the diplexer enables full-duplex operation for Ku-band SATCOM on-the-move systems, effectively separating the [11.7-12.75] GHz downlink (Rx) band from the [13.7514.50] GHz uplink ( $\mathrm{Tx}$ ) band, yielding an isolation larger than $20 \mathrm{~dB}$ and an insertion loss below $3.7 \mathrm{~dB}$ over a fractional bandwidth (FBW) of $8.6 \%$ in $\mathrm{Rx}$ and $5.3 \%$ in $\mathrm{Tx}$.

Index Terms-Dual-mode cavity filter, Ku-band Diplexer, SATCOM-on-the-move, Substrate integrated waveguide.
\end{abstract}

\section{INTRODUCTION}

Satellite communication (SATCOM)-on-the-move (SOTM) enables $5 \mathrm{G}$ wireless communication in the most remote locations on the planet as in the air. To exploit the full capacity of the data link with the satellites, full-duplex operation, with simultaneous uplink and downlink communication, is preferred. For easy deployment in mobile user equipment, a digitally controllable [1] shared-aperture phased antenna array that covers both uplink and downlink bands should be compact, lightweight and integrable. Within a footprint that is limited by the maximally allowed antenna element spacing, highly specialized components must be fitted, such as dual-band antennas, integrated circuits, feed lines, transitions and diplexers [2]-[7]. The latter are of utmost importance to implement full-duplex communication in a shared aperture system with minimal interference between transmit and receive chains. To further limit undesired interaction with the environment or other system components, the diplexer should also be self-packaged, while being tightly and compactly integrated with the antenna and the active beamforming electronics.

Most existing diplexer designs, which combine two filters by a T-junction [8]-[14], exhibit excellent performance, but are bulky and non scalable. Other diplexer types rely on mode perturbation [10]-[12], negative coupling and modal crosscoupling [9], [13]. The latter also plays a role in common dualmode cavity-based designs, which is the topology exploited in this contribution. Other solutions take advantage of special transitions with diplexer functionality [6] or implement a selfdiplexing antenna [15]. However, these designs yield limited bandwidths and their geometry does not allow scalability. Also more specialized and complex designs exist [16]-[21]. How- ever, they lack scalability and introduce too much complexity for the application of interest. The scalable diplexers in [22] make use of technologies that are too expensive. An interesting approach to reduce the diplexer's size in a more flexible way consists in exploiting a common cavity that replaces the Tjunction [23]-[28].

This contribution presents a novel very compact and costeffective Substrate-Integrated-Waveguide (SIW) diplexer architecture, targeting SOTM applications in the Ku-band. Therefore, the designed single-layer diplexer separates the [11.7-12.75] GHz downlink (Rx) band from the [13.7514.50] $\mathrm{GHz}$ (Tx) uplink band, with an isolation larger than $20 \mathrm{~dB}$ and an insertion loss lower than $3.7 \mathrm{~dB}$. The fractional bandwidths (FBWs) in which this performance is obtained correspond to $8.6 \%$ for $\mathrm{Rx}$ and $5.3 \%$ for Tx, respectively. In particular, the FBW along the Rx path is large in comparison to existing diplexer designs. This response is achieved by first dimensioning three substrate integrated rectangular cavities (SIRC) as to achieve a third-order Chebyshev filter characteristic, for both separate Tx and Rx filters. Next, a common dual-mode cavity is exploited to integrate both circuits into a single-layer diplexer.

\section{KU-BAND SIW DIPLEXER DESIGN}

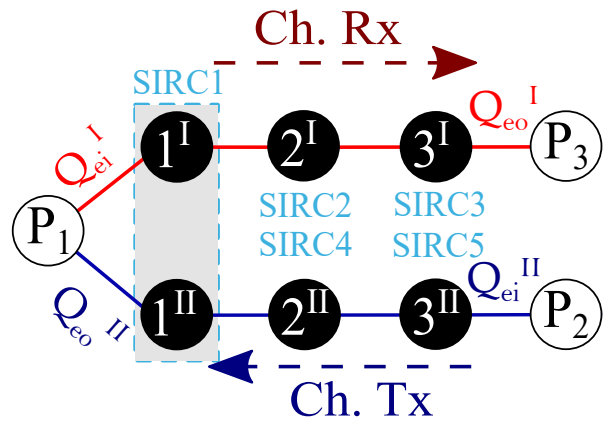

Fig. 1. Topology of single-layer third-order direct-coupled SIW diplexer.

The topology of the diplexer is schematically shown in Fig. 1. The $\mathrm{Rx}$ and $\mathrm{Tx}$ channel, annotated by $I$ and $I I$ superscripts, respectively, both consist of three SIRC resonators, implemented in a $0.508 \mathrm{~mm}$-thick Rogers RO4350B $\left(\epsilon_{r}=3.66, \tan \delta=0.0037\right)$ high-frequency laminate. Two cavities operate with their $T E_{101}$ resonance at the center frequency $(\mathrm{CF})$ of the corresponding channel, being $f^{I}$ or $f^{I I}$. The third cavity is shared between both channels and is designed such that its $T E_{101}$ resonance corresponds to $f^{I}$ 


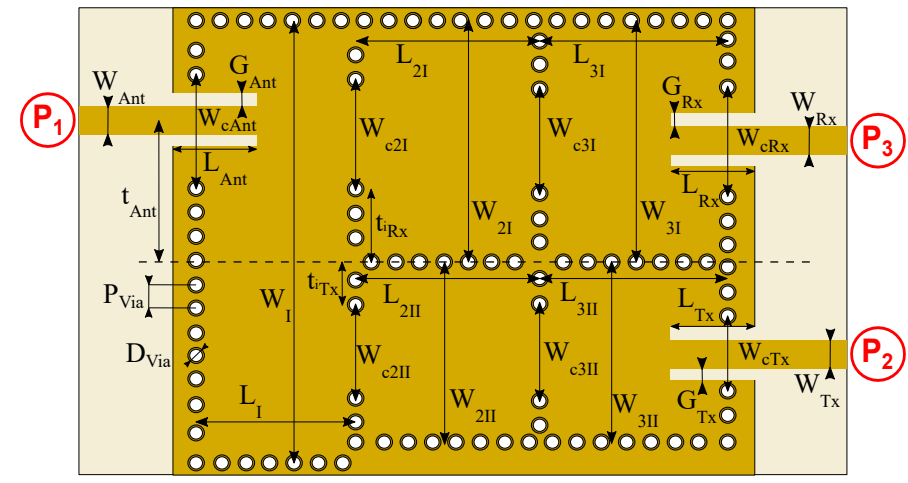

Fig. 2. Geometric configuration of single-layer third-order direct-coupled SIW diplexer. Optimized dimensions in millimeters: $D_{V i a}=0.5, G_{A n t}=0.6$, $G_{R x}=0.51, G_{T x}=0.53, L_{2 I}=7.49, L_{2 I I}=7.77, L_{3 I}=7.54$ $L_{3 I I}=6.92, L_{A n t}=3.45, L_{I}=6.5, L_{R x}=1.93, L_{T x}=2.75$ $P_{V i a}=1, t_{A n t}=5.77, t_{i_{R x}}=3, t_{i_{T x}}=1.93, W_{2 I}=10.47, W_{2 I I}=$ 7.76, $W_{3 I}=10.47, W_{3 I I}=8.78, W_{A n t}=1.4, W_{c 2 I}=4.74, W_{c 2 I I}=$ $3.9, W_{c 3 I}=4.48, W_{c 3 I I}=4.05, W_{c A n t}=5.74, W_{c R x}=4.6, W_{c T x}=$ $3.46, W_{I}=18.97, W_{R x}=1.4, W_{T x}=1.4, h_{\text {dielectric }}=0.508$. Singlelayer dielectric implemented in Rogers 4350B $\left(\epsilon_{r}=3.66, \tan \delta=0.0037\right)$.

and the $T E_{201}$ resonance to $f^{I I}$, thus operating as a dualmode cavity. The coupling to adjacent resonators and the feed of the common cavity is optimized through modal cross (bypass) coupling [29] to generate an additional transmission zero between the Tx and Rx channel, allowing to achieve the requirements with a third-order Chebyshev-like diplexer design.

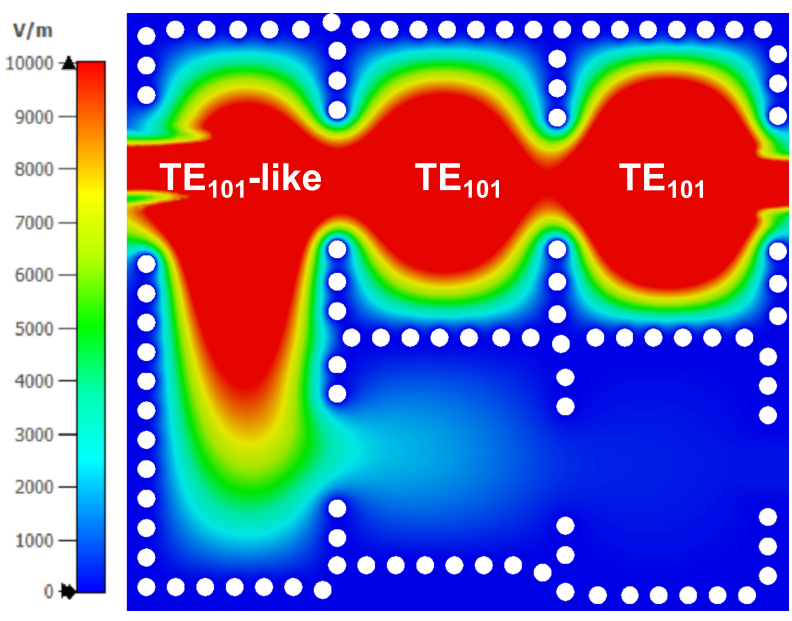

Fig. 3. Electric field magnitude distributions at $12.2 \mathrm{GHz}$ when port 1 (antenna) is excited. Signal propagating to port 3 ( $\mathrm{Rx}$, receiver).

Fig. 2 shows the final diplexer design, featuring a footprint of $22.1 \mathrm{~mm}$ by $19.7 \mathrm{~mm}$. All relevant dimensions, obtained by computer-aided optimization in CST Microwave Studio, are listed in the figure's caption. The operating principle of the realized diplexer is illustrated in Fig. 3, showing the signal at $12.2 \mathrm{GHz}$, propagating from the antenna to the receiver port and being blocked by the dual-mode cavity to suppress potential reception at the transmitter port, and in Fig. 4, visualizing the signal at $14.4 \mathrm{GHz}$, propagating from

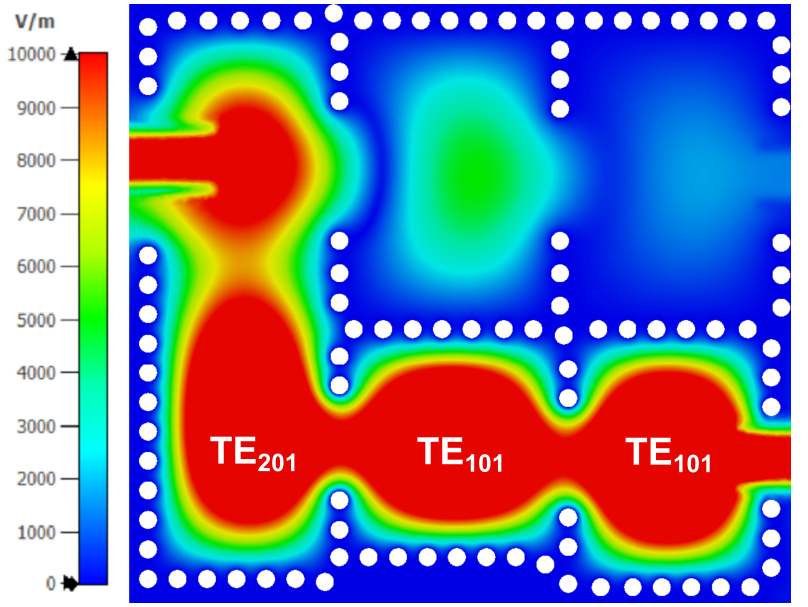

Fig. 4. Electric field magnitude distributions at $14.1 \mathrm{GHz}$ when port 2 (Tx, transmitter) is excited. Signal propagating to port 1 (antenna).

the transmitter port to the antenna and suppressing potential reception at the receiver port by the dual-mode cavity and the transmission zero implemented by modal cross-coupling.

\section{PROTOTYPE AND MEASUREMENTS}

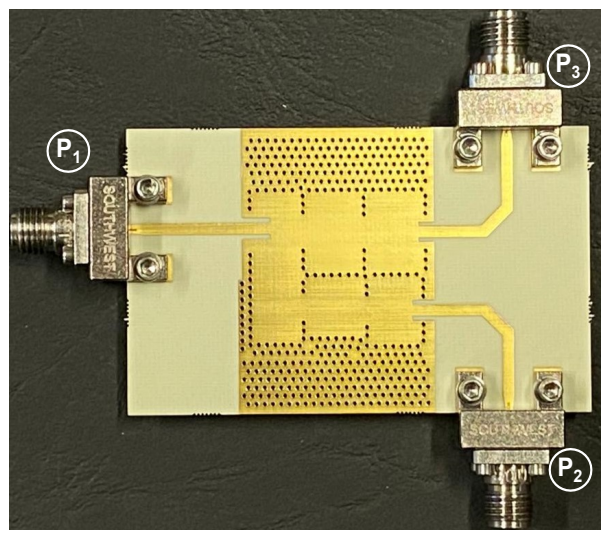

Fig. 5. Fabricated prototype of the single-layer third-order direct-coupled diplexer.

To validate its operation, a prototype of the diplexer, shown in Fig. 5, was manufactured and characterized. Fig. 6 shows the transmission and reflection coefficients $\left(S_{31}\right.$ and $\left.S_{33}\right)$ along the Rx path, demonstrating that the [11.7-12.75] GHz band is covered with a maximum insertion loss of $3.69 \mathrm{~dB}$, and with more than $20.47 \mathrm{~dB}$ suppression in the Tx band. Fig. 7 confirms that the transmission coefficient $S_{12}$ along the Tx path exhibits an insertion loss lower than $3.59 \mathrm{~dB}$ over the complete [13.75-14.5] GHz band, with more than $25.18 \mathrm{~dB}$ suppression in the Rx band. Moreover, the isolation between $\mathrm{Rx}$ and Tx channel was measured to be better than $20.57 \mathrm{~dB}$.

\section{CONCLUSION}

A single-layer third-order direct-coupled SIW diplexer is proposed, with a minimum isolation of $20 \mathrm{~dB}$ and an insertion 


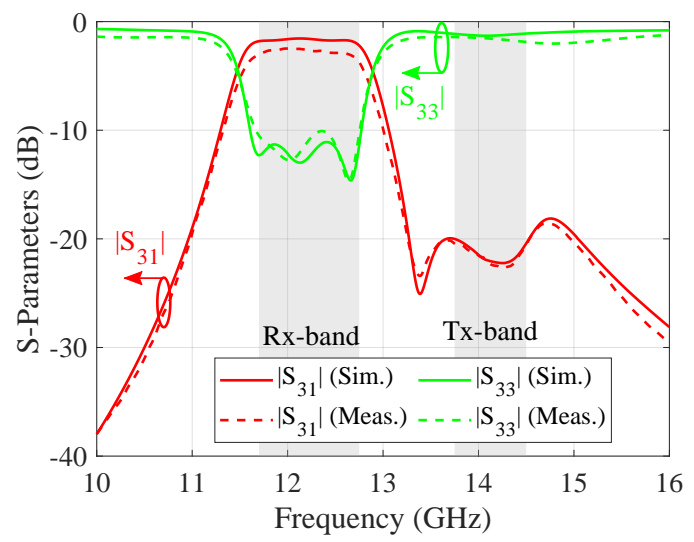

Fig. 6. Simulated (full line) versus measured (dashed line) transmission $\left(S_{31}\right)$ and reflection $\left(S_{33}\right)$ coefficients along the Rx path.

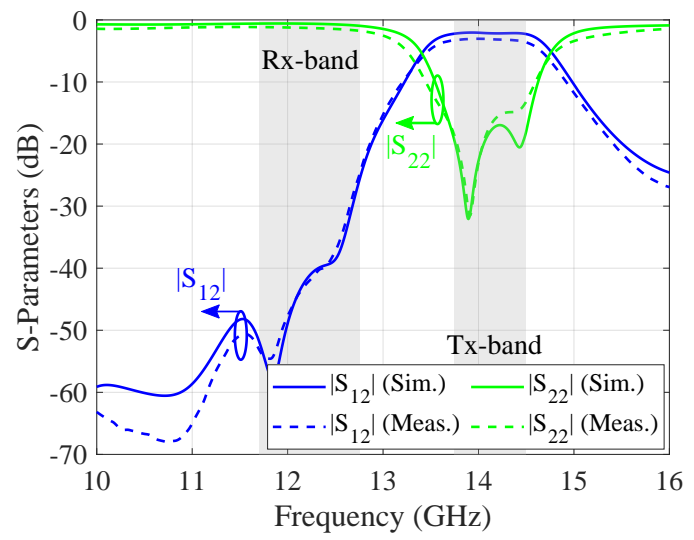

Fig. 7. Simulated (full line) versus measured (dashed line) transmission $\left(S_{12}\right)$ and reflection $\left(S_{22}\right)$ coefficients along the Tx path.

loss below $3.7 \mathrm{~dB}$, paving the way for low-cost sharedaperture full-duplex antenna arrays for next-generation $\mathrm{Ku}-$ band SATCOM-on-the-move applications.

\section{REFERENCES}

[1] J. S. Herd and D. M. Convay, "The evolution to modern phased array architectures," Proc. IEEE, vol. 104, no. 3, pp. 519-529, Mar 2016.

[2] T.-Y. Yun et al., "A 10 to 21-GHz, low-cost, multifrequency, and fullduplex phased-array antenna system," IEEE Trans. Antennas Propag., vol. 50, no. 5, pp. 641-650, 2002.

[3] C. T. Rodenbeck et al., "Ultra-wideband low-cost phased-array radars," IEEE Trans. Microw. Theory Tech., vol. 53, no. 12, pp. 3697-3703, 2005.

[4] S. Hong et al., "A multiband, compact, and full-duplex beam scanning antenna transceiver system operating from 10 to $35 \mathrm{GHz}$," IEEE Trans. Antennas Propag., vol. 54, no. 2, pp. 359-367, 2006.

[5] A. Vosoogh et al., "An integrated Ka-band diplexer-antenna array module based on gap waveguide technology with simple mechanical assembly and no electrical contact requirements," IEEE Trans. Microw. Theory Tech., vol. 66, no. 2, pp. 962-972, 2018.

[6] K. Erkelenz et al., "A compact K-/Ka-band rectangular-to-coplanar waveguide transition with integrated diplexer," IEEE Microw. Guided Wave Lett., vol. 31, no. 6, pp. 642-645, 2021.

[7] G. Gültepe et al., "A 1024-element Ku-band SATCOM dual-polarized receiver with $>10-\mathrm{dB} / \mathrm{K}$ G/T and embedded transmit rejection filter," IEEE Trans. Microw. Theory Tech., pp. 1-1, 2021.
[8] H. J. Tang, W. Hong, J.-X. Chen, G. Q. Luo, and K. Wu, "Development of millimeter-wave planar diplexers based on complementary characters of dual-mode substrate integrated waveguide filters with circular and elliptic cavities," IEEE Trans. Microw. Theory Tech., vol. 55, no. 4, pp. 776-782, 2007.

[9] S. Sirci, J. D. Martínez, J. Vague, and V. E. Boria, "Substrate integrated waveguide diplexer based on circular triplet combline filters," IEEE Microw. Guided Wave Lett., vol. 25, no. 7, pp. 430-432, 2015.

[10] P. Chu, W. Hong, M. Tuo, K.-L. Zheng, W.-W. Yang, F. Xu, and K. Wu, "Dual-mode substrate integrated waveguide filter with flexible response," IEEE Trans. Microw. Theory Tech., vol. 65, no. 3, pp. 824-830, 2017.

[11] K. Song, Y. Zhou, Y. Chen, A. Mohamed Iman, S. Richard Patience, and Y. Fan, "High-isolation diplexer with high frequency selectivity using substrate integrate waveguide dual-mode resonator," IEEE Access, vol. 7, pp. 116676-116683, 2019.

[12] F. Cheng, C. Gu, B. Zhang, Y. Yang, and K. Huang, "High isolation substrate integrated waveguide diplexer with flexible transmission zeros," IEEE Microw. Guided Wave Lett., vol. 30, no. 11, pp. 1029-1032, 2020.

[13] P.-L. Chi, H.-T. Shih, and T. Yang, "5G millimeter-wave substrateintegrated waveguide quad-channel diplexer with high in-band and wideband isolation," IEEE Microw. Guided Wave Lett., vol. 31, no. 6, pp. $650-653,2021$.

[14] Z. Su et al., "High-isolation and wide-stopband SIW diplexer using mixed electric and magnetic coupling," IEEE Trans. Circuits Syst. II, vol. 67, no. 1, pp. 32-36, 2020.

[15] A. A. Khan and M. K. Mandal, "Compact self-diplexing antenna using dual-mode SIW square cavity," IEEE Antennas Wireless Propag. Lett., vol. 18, no. 2, pp. 343-347, 2019.

[16] Y. Dong and T. Itoh, "Substrate integrated waveguide loaded by complementary split-ring resonators for miniaturized diplexer design," IEEE Microw. Guided Wave Lett., vol. 21, no. 1, pp. 10-12, 2011.

[17] F. Cheng, X. Lin, K. Song, Y. Jiang, and Y. Fan, "Compact diplexer with high isolation using the dual-mode substrate integrated waveguide resonator," IEEE Microw. Guided Wave Lett., vol. 23, no. 9, pp. 459$461,2013$.

[18] H. Oraizi and E. Bidaki, "Optimum design of a miniaturized multilayer SIW diplexer using orthogonal-mode resonator," in 7'th IST, 2014, pp. 274-277.

[19] M. F. Hagag, M. Abu Khater, M. D. Hickle, and D. Peroulis, "Tunable SIW cavity-based dual-mode diplexers with various single-ended and balanced ports," IEEE Trans. Microw. Theory Tech., vol. 66, no. 3, pp. $1238-1248,2018$.

[20] A. Iqbal, J. J. Tiang, C. K. Lee, and B. M. Lee, "Tunable substrate integrated waveguide diplexer with high isolation and wide stopband," IEEE Microw. Guided Wave Lett., vol. 29, no. 7, pp. 456-458, 2019.

[21] I. Llamas-Garro, F. Mira, P. Zheng, Z. Liu, L.-S. Wu, and Y. Wang, "All resonator based LTCC diplexer using substrate integrated waveguides," Electron. Lett., vol. 53, 092017.

[22] A. Sieganschin, T. Jaschke, H. K. Mitto, N. J. Lamann, J. Waldhelm, and A. F. Jacob, "A compact low-loss multilayer SIW diplexer at K/Kaband," in $2019 \mathrm{GeMiC}, 2019$, pp. 51-54.

[23] A. García-Lampérez, M. Salazar-Palma, and S. H. Yeung, "SIW compact diplexer," in 2014 IEEE MTT-S IMS, 2014, pp. 1-4.

[24] K. Zhou, C.-X. Zhou, and W. Wu, "Compact SIW diplexer with flexibly allocated bandwidths using common dual-mode cavities," IEEE Microw. Guided Wave Lett., vol. 28, no. 4, pp. 317-319, 2018.

[25] H.-W. Xie, K. Zhou, C.-X. Zhou, and W. Wu, "Compact SIW diplexers and dual-band bandpass filter with wide-stopband performances," IEEE Trans. Circuits Syst. II, vol. 67, no. 12, pp. 2933-2937, 2020.

[26] Z. Kordiboroujeni and J. Bornemann, "Substrate integrated waveguide diplexer with dual-mode junction cavity," in EuMC, 2015, pp. 753-756.

[27] K. Zhou, C. Zhou, and W. Wu, "Compact planar substrate-integrated waveguide diplexers with wide-stopband characteristics," Int. Journ. RF and Microw. Comp.-Aided Eng., vol. 30, no. 6, p. e22179, 2020.

[28] W.-L. Tsai, T.-M. Shen, B.-J. Chen, T.-Y. Huang, and R.-B. Wu, "Design of single-branch laminated waveguide diplexers using modal orthogonality," IEEE Trans. Microw. Theory Tech., vol. 61, no. 12, pp. 4079-4089, 2013

[29] S. Amari and U. Rosenberg, "Characteristics of cross (bypass) coupling through higher/lower order modes and their applications in elliptic filter design," IEEE Trans. Microw. Theory Tech., vol. 53, no. 10, pp. 3135$3141,2005$. 